Do Social Features Help in Video-Centric Online Learning Platforms?

A Social Presence Perspective

\author{
Stephanie A. Andel \\ Corresponding Author \\ sandel@iu.edu \\ Triparna de Vreede \\ tdevreede@usf.edu \\ Paul E. Spector \\ pspector@usf.edu \\ Balaji Padmanabhan \\ bp@usf.edu \\ Vivek K. Singh \\ vsingh@umsl.edu \\ Gert-Jan de Vreede \\ gdevreede@usf.edu \\ andiana University-Purdue University Indianapolis \\ 402 N. Blackford Street \\ Indianapolis, IN 46202 \\ ${ }^{\mathrm{b}}$ The University of South Florida \\ 4202 E. Fowler Ave. \\ Tampa, FL 33620 \\ ${ }^{\mathrm{c}}$ The University of Missouri - St. Louis \\ 1 University Blvd. \\ St. Louis, MO 63121
}

Partial support for this research was provided by onQ.

This is the author's manuscript of the work published in final edited form as:

Andel, S. A., de Vreede, T., Spector, P. E., Padmanabhan, B., Singh, V. K., \& de Vreede, G.-J. (2020). Do social features help in video-centric online learning platforms? A social presence perspective. Computers in Human Behavior, 113, 106505. https://doi.org/10.1016/j.chb.2020.106505 


\begin{abstract}
The popularity of online learning is growing exponentially. Accordingly, it is critical to understand how we can facilitate the learning experience for users in these unique online environments. Past research consistently finds that one important antecedent of online learning outcomes is social presence. However, there is limited research related to how to foster social presence perceptions among users. Further, research has yet to understand whether there are boundary conditions for the positive effects of social presence perceptions on user outcomes (e.g., perceived learning, satisfaction). The current work describes two studies to address these questions. The first study uses an experimental design to investigate how the perception of social presence can be increased in the online learning context. In the second study, we investigate the degree to which social presence perceptions are more beneficial for some individuals versus others. Specifically, we consider two personality traits (i.e., conscientiousness, extraversion) as moderators of the relationships between social presence perceptions and two outcomes perceived learning and satisfaction. Overall, the results of these two studies provide important insights into how to enhance user outcomes in online learning and training environments.

Keywords: online learning, social presence, social features, personality
\end{abstract}




\section{Do Social Features Help in Video-Centric Online Learning Platforms?}

\section{A Social Presence Perspective}

\section{Introduction}

As technology continues to advance, so too does the landscape of the education system, with the traditional classroom frequently being replaced with online learning environments. In fact, distance education enrollment has been steadily increasing over the years, with the percentage of U.S. college students who are enrolled in at least one distance education course rising from $25.9 \%$ in 2012 to $31.6 \%$ in 2016 (Seaman, Allen, \& Seaman, 2018). This increase to online learning has grown exponentially in recent months due to the coronavirus (COVID-19) pandemic, with an unprecedented number of primary, secondary, and postsecondary courses being swiftly transformed from a traditional to an online format in order to reduce physical contact (Burke, 2020; Goldstein, 2020). Such an abrupt transition to online learning has led to several challenges for instructors and students alike. In fact, a recent survey conducted by Barnes \& Noble College Insights surveyed 432 college students across the United States about their expectations for moving from traditional classrooms to online courses in the wake of the COVID-19 pandemic. The majority of respondents noted various concerns about the transition. For instance, $64 \%$ of respondents cited concerns about remaining focused and disciplined in an online format, and 55\% reported concerns that they would be negatively impacted by lack of social interaction in the online learning environment (Brown, 2020). Accordingly, is it arguably more important now than ever to empirically assess and understand how to effectively optimize and enhance the online learning experience.

Within the existing online learning literature, there is a general consensus that social presence, or the ability to perceive others in a mediated environment (Richardson, Maeda, Lv, \& 
Caskurlu, 2017), is an important factor to cultivate within the online classroom. In fact, social presence has been consistently linked to numerous positive outcomes. For instance, recent metaanalytic evidence from Richardson et al. (2017) shows that when online students perceive greater social presence within their online classrooms, they experience greater satisfaction $(\rho=.56)$ and perceived learning $(\rho=.51)$. Other research has shown that social presence fosters intentions to take future online courses (Reio \& Crim, 2013), persistence (e.g., Berger \& Milem, 1999), motivation to participate (e.g., Swan \& Shih, 2005; Mazzolini \& Maddison, 2007), and student performance (e.g., Hughes, Luo, Kwok, \& Loyd, 2008). These findings have major implications for large scale online learning environments where instructors are searching for tangible ways to maximize and enhance the online learning experience.

The aforementioned literature highlights the need for educators to understand how to optimize opportunities for social presence within the online classroom. As a result, a number of researchers have investigated ways to increase social presence perceptions in an online learning context. Much of this research has focused upon increasing social presence through the utilization of features such as the use of personal profiles (Kear, Chetwynd, \& Jefferis, 2014), text messages (DuVall, Powell, Hodge, \& Ellis, 2007), individualized video feedback (Lowenthal \& Dunlap, 2011), and one-on-one email communication (Dunlap \& Lowenthal, 2010). While insights gained from this previous research is informative, existing work focuses almost exclusively upon ways to enhance instructor social presence, and does not considers ways to increase user social presence. Further, existing research caters to online classroom environments that allow for individualized and small-group interactions among students and teachers. However, many online learning environments cannot feasibly incorporate one-on-one or small group interactions. As a result, many online instructors rely primarily upon video to 
disseminate class content (Guo \& Reinecke, 2014), and therefore have limited means to establish social presence, especially among users. Therefore, it is important to consider how to leverage social presence in such video-centric online environments, where personal, one-on-one communications or small group work are not feasible.

Additionally, we have yet to understand the boundary conditions for the impact of social presence on learning outcomes. Specifically, there is a dearth of research that investigates whether social presence benefits some students more so than others. This is an area that is worthy of investigation, because in order to truly understand the impact of social presence on the learners' experience, it is imperative know which individuals gain the most from social presence. As noted by Lowenthal and Mulder (2017), “...people perceive social presence differently and each person has different social presence needs. Therefore, one strategy might not work equally for all learners" (p. 40). Taking this individualized approach into consideration, we examine the role of personality as a moderator of the relationships between social presence and user outcomes (i.e., perceived learning and user satisfaction).

Therefore, the goals of the current research are to investigate the impact of social features on social presence perceptions (Study 1), and to understand the impact of user personality on the relationship between social presence and learner outcomes (i.e., satisfaction, perceived learning; Study 2). To address these questions, we examine the effectiveness of social features within an online learning platform called onQ. This platform allows users to asynchronously communicate by inserting comments and emoji reactions along the timeline of the video. 


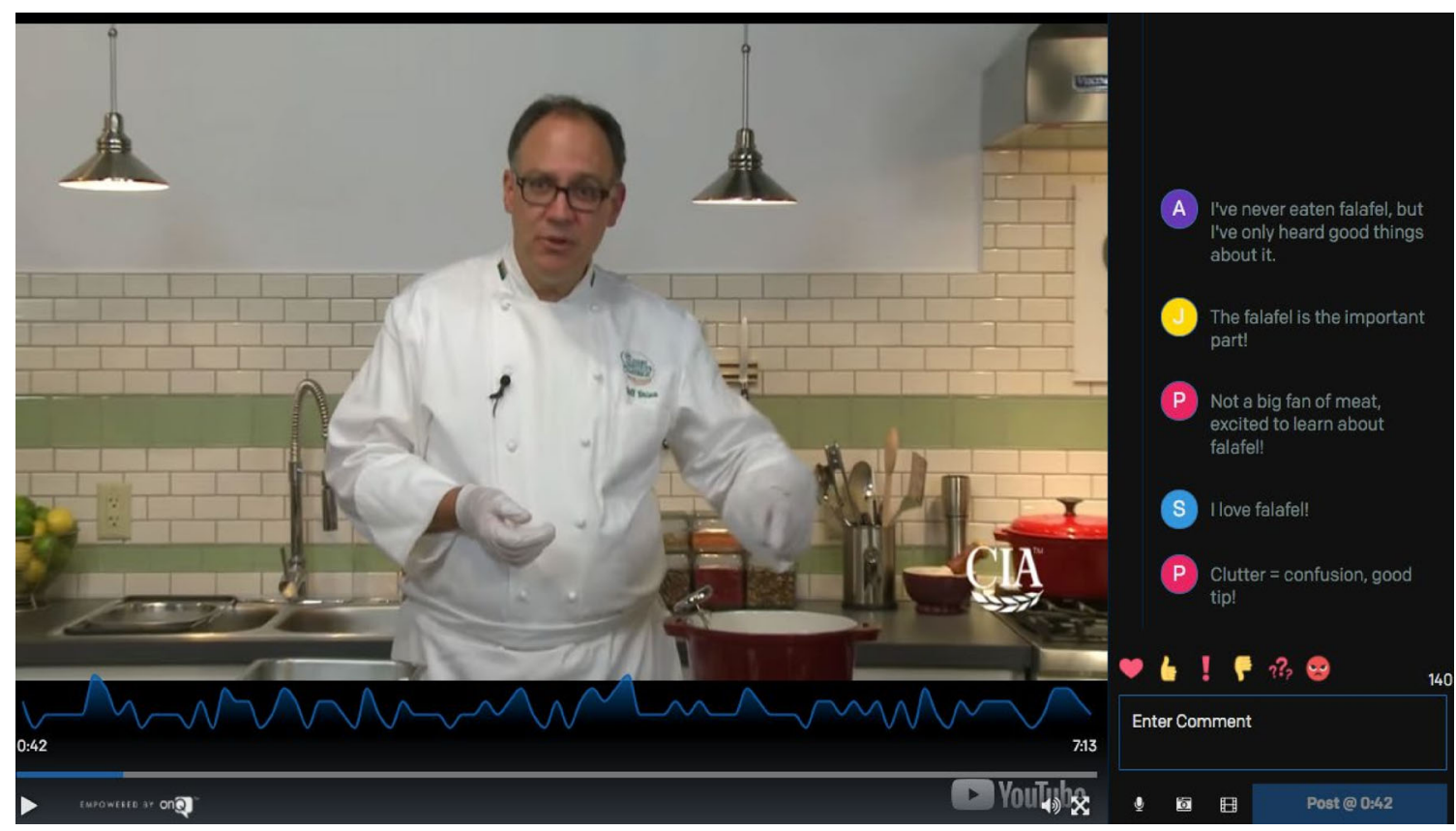

Figure 1. A screenshot of the onQ platform with seeded comments.

\section{Literature Review}

\subsection{Social Presence in Online Learning}

Research on social presence has a rich history, stemming from its original conceptualization by Short, Williams, and Christie (1976), who proposed that social presence was an integral quality of the communication medium. This early perspective assumed that online communication media "filtered out" important audio and visual cues inherent in face to face communication, and therefore were less personal and incapable of fostering high degrees of social presence. According to this perspective, online learning environments have inherently less social presence than traditional face-to-face classrooms. Over time, however, perceptions on social presence have changed, largely due to researchers such as Gunawardena (1995) and Garrison et al. (1999), who claimed that individuals can cultivate social presence by "project[ing] their personal characteristics into the community, thereby presenting themselves to the other participants as "real people"' (Garrison et al., 1999, p. 89). This notion that social 
presence can be cultivated and fostered has enjoyed steady support, as researchers have shown that individuals are in fact able to convey their personalities and connect with others via online communication media, such as text-based communication (e.g., Swan, 2003; Swan \& Shih, 2005). This research provides evidence that social presence is not as simple as originally assumed as individuals in online environments are able to "make up" for lost social cues in mediated environments. For instance, studies have shown that displaying humor and using emoticons to express emotions can influence online social presence perceptions (Rourke, Anderson, Garrison, \& Archer, 2001; Swan, 2003).

As a result, recent research has begun to investigate the effectiveness of adding social features to online learning environments in order to facilitate perceptions of social presence among learners. For instance, research by DuVall et al. (2007) and Dunlap \& Lowenthal (2010) found that one-on-one communications between students and their instructor can increase perceptions of instructor social presence. Additionally, Kear and colleagues (2014) showed that the addition of personal profiles and photographs in an online environment was linked with connection among learners, while research by Swan (2003) demonstrated that online discussions via discussion boards may increase perceptions of social presence. These research studies show that, when provided with additional avenues to connect and interact, users are more likely to feel a greater sense of belongingness and ultimately perceive higher levels of presence within the online classroom.

\subsection{Social Presence in a Video Context}

As video is a central component of the online learning experience, there exists some (albeit limited) research studies that consider ways to increase social presence within videospecific online learning contexts. For example, Borup, West, and Graham (2012) found evidence 
that the addition of asynchronous videos in an online classroom was effective at enhancing perceptions of instructor (but not student) social presence. Further, Lyons, Reysen, and Pierce (2012) found that when online lectures included videos of the instructor, students perceived higher levels of instructor social presence. Additionally, a research study by Borup, West, Thomas, and Graham (2014) compared the efficacy of video feedback versus text feedback on perceptions of instructor social presence. However, these techniques are not amenable to large scale environments, and generally focus on using video to enhance instructor (versus user) social presence. In fact, to our knowledge, no existing research has investigated feasible ways to enhance social presence among users in a video-centric environment.

In this research we leverage access to a unique platform that provides users the opportunity comment and share emojis along the timeline of an online video. We propose that the incorporation of social opportunities along the timeline of an online video can provide a feasible means to increase social presence perceptions among users in a video-centric environment. In this platform users are able to asynchronously add video-tagged comments and emojis as they watch an online video. These comments and emojis will be seen by the subsequent users that watch the video, who will be able to add their own comments/emojis and respond to others. In the current study, we focus specifically upon the impact of video-tagged comments on social presence perceptions. Ultimately, we postulate that the addition of this social feature along the video timeline will increase social presence perceptions among users.

Hypothesis 1: User comments that appear along the timeline of an online learning video will lead to higher social presence perceptions among users. 


\section{Study 1}

The purpose of study 1 was to test the impact of asynchronous user comments on user social presence perceptions. This study was conducted within the onQ learning platform, which is described in detail in Section 2.1.3. This study was a controlled experiment, and we compared a control condition, in which there were no comments from other users, with an experimental condition, in which participants could see comments from other users (seeded by the research team).

\subsection{Study 1 Method}

\subsubsection{Participants}

Seventy-one participants were recruited through Amazon's Mechanical Turk (MTurk). Inclusion criteria were that individuals must be from the United States, must have access to a laptop or desktop computer, have at least a 95\% approval rating within the MTurk system, and have completed at least 100 MTurk HITs in the past. The average age was 34.92 and $29.6 \%$ of the sample was female. In terms of education, $83.1 \%$ of the sample had at least some college. Overall, 36 individuals were in the control (unseeded) condition and 35 were in the experimental (seeded) condition.

\subsubsection{Procedure}

Upon agreement to participate, participants were asked to watch a brief 7-minute instructional video about cooking. Individuals were told that they were a part of a large group who would be watching the online video (in reality, each individual received their own individual link). Immediately following the video, participants completed a brief survey about their online video experience, which included a measure of social presence. In exchange for their participation, each participant was compensated with $\$ 2.00$ through the MTurk system. 
During the experiment, each individual was randomly assigned into one of two conditions. In the first condition (i.e., control), participants watched the video via an embedded video platform with commenting and emoji functionality. However, no comments from other users appeared. In the second condition (i.e., experimental), participants watched the video via the same video platform with commenting and emoji functionality. In this condition, users saw comments appear from other users throughout the duration of the video.

\subsubsection{Online Learning Platform and Seeding Procedure}

The online learning platform used in the current study was onQ, which is a platform that emphasizes social learning through the incorporation of social features. Specifically, the platform creates an overlay on top of any video content, in which a field with commenting and emoji features appear to the right of the video. Users are then able to use these commenting and emoji features to asynchronously communicate with other users. Each time a user logs in to view the video, they will see (and have the opportunity to respond to) the existing comments and emojis from others. For the current study, the cooking video was embedded within the onQ player (see Figure 1). Within the control condition, all users had the opportunity to comment and share emojis, but no comments from other users appeared. Within the experimental condition, the users had the same opportunity to comment and share emojis, but there were also comments from other users that appeared at various intervals as the video progressed.

In a traditional onQ experience, all users have access to the same video link in which the comments build over time, as each user can see and add to the existing comments. However, in order to keep the comments consistent across users within the experimental condition in the current study, we provided each user with an individual link in which we seeded the same previous user comments. In order to acquire realistic user comments to seed into these video 
links for the experimental condition, we asked five graduate students (who were not involved in the development of the study) to watch the video in the onQ platform and to provide comments while watching. These comments were recorded and inserted into the individual video links for each person in the experimental condition, but for the control condition links. Ultimately, our seeding process ensured that the comments were generated organically, and that every study participant in the experimental condition viewed the same comments.

\subsubsection{Survey Measures}

Social presence was measured with six items, four of which were adapted from existing social presence scales (i.e., Bailenson, Blascovich, Beall, \& Loomis, 2003; Kreijns, Kirschner, Jochems, \& van Buuren, 2011) and two of which were developed for the current study. Participants rated the degree to which they agreed with each item $(1=$ Strongly disagree; $5=$ Strongly agree). Example items are "The other users in the online environment seemed to be conscious and alive to me", "I was aware of other users in this online environment", and "I felt like I was in the presence of other users in the online environment". Cronbach's alpha $(\alpha)$ for this scale was .95 .

\subsection{Study 1 Results}

An independent-samples t-test was conducted to investigate the difference in social presence perceptions across the two conditions. In support of Hypothesis 1, results showed that there was a significant difference between the groups on social presence perceptions, $t=4.53, p$ $<.001$. Specifically, individuals within the experimental (seeded) condition $(M=3.53, S D=$ 1.21) had significantly higher perceptions of social presence than the control condition $(M=$ $2.23, S D=1.21$ ). The standardized mean difference (i.e., Cohen's $d$ ) was 1.07 , which according to Cohen's (1988) guidelines is considered to be a large effect size. The $95 \%$ confidence interval 
for $d=1.07$ is $[0.53,1.59]$, indicating that there is a .95 probability that the true standardized mean difference $(d)$ falls between 0.53 and 1.59 .

In order to assess if these differences may be due to group mean differences related to the video topic (cooking), we conducted a one-way analysis of covariance (ANCOVA) to see if the group mean differences in social presence held while controlling for prior cooking experience (all participants were asked to respond to the item, "Before this study, how much experience did you have with cooking" on a scale of $1=$ None to $5=A$ lot ). Results of this analysis showed that the condition (i.e., seeded vs. unseeded) remained significantly associated with social presence perceptions, even after controlling for prior cooking experience, $F(1,68)=20.89, p<.01$. These results provide further evidence that the differences in social presence were due to the comment seeding manipulation.

\subsection{Study 1 Brief Discussion}

The aim of this initial study was to assess if features of a short-term, video centric online learning environment influence perceptions of social presence among users. More specifically, this study provides evidence that user comments that appear along the duration of an online video are significantly related to increased users' perceptions of social presence. These results have important implications for large-scale online learning environments by providing a tangible way to increase social presence perceptions in these video-centric environments.

Building upon these results, we conducted a second study to explore whether the effects of social presence perceptions vary across individuals. Specifically, we were interested in exploring the possibility that social presence may be beneficial for some individuals more than others. We tested this question in Study 2 with a correlational design in order to investigate a more natural situation within the onQ platform. 


\section{Study 2 Overview and Hypotheses}

Building upon the results of Study 1, the purpose of Study 2 was to understand who benefits most from perceiving high degree of social presence. To explore this question, we examine user personality factors as moderators of the relationships between social presence perceptions and two outcomes, namely perceived learning and satisfaction. These outcomes were chosen for a couple of reasons. First, these outcomes reflect the components of a traditional reaction-level course evaluation that aligns with Kirkpatrick's (1959) commonly cited evaluation guidelines, with user satisfaction reflecting one's affective reaction to the learning experience (i.e., the degree to which one enjoyed the online experience), and with perceived learning reflecting one's utility reaction to the learning experience (i.e., the degree to which one felt that the online experience was useful). Second, satisfaction and perceived learning serve as the two most commonly examined variables in relation to social presence, and in fact a recent metaanalysis by Richardson et al. (2017) focused specifically on these outcomes and demonstrated the robustness of their relationships with social presence.

Building upon this existing literature, we investigate whether certain personality traits will moderate the degree to which social presence perceptions are linked to users' satisfaction and perceived learning. In particular, we consider the moderating role of two personality traits: extraversion and conscientiousness. We chose to examine these personality traits in order to capture one trait that is associated with social needs and tendencies (i.e., extraversion), and one trait that is associated with learning and achievement (i.e., consciousness).

Extraversion is defined as the tendency to prefer social activities (Costa \& McCrae, 1992). We propose that extraverted individuals will feel more satisfied and learn more in an online environment where they perceive the presence of other learners. This is because extraverts 
have an inherent need for social connection (Watson \& Clark, 1997) and therefore when they are in environments where they perceive high levels of social presence, they will fulfill their social needs, and in turn will be more satisfied and learn more within the learning environment. Alternatively, when individuals are low in extraversion (i.e., introverted), they may prefer less social presence. That is, they will not draw energy from socially connecting with others, and therefore they will likely prefer an online environment where they can simply focus on learning the material without the opportunity for social connection.

Hypothesis 1: Extraversion will moderate the relationship between social presence and (a) perceived learning, and (b) satisfaction, such that these relationships will be stronger when extraversion is high (versus low).

Conscientiousness is defined as the tendency to be competent, hard-working, dependable, and achievement oriented (Costa \& McCrae, 1992). This personality trait is the strongest and most stable personality predictor of learning (Trapmann, Hell, Hirn, \& Schuler, 2007). We posit that when individuals are high in conscientiousness, social presence will not influence their satisfaction or perceived learning. This is because these individuals are inherently self-directed and intrinsically motivated (Komarraju, Karau, \& Schmeck, 2009), and therefore they are likely to proactively engage with the online learning environment, regardless of external factors such as social presence. However, when individuals are low in conscientiousness, they may need other external factors to motivate them to engage with the learning environment. In this case, high levels of social presence may serve to motivate these individuals to engage, leading to higher levels of perceived learning and satisfaction. 
Hypothesis 2: Conscientiousness will moderate the relationship between social presence and (a) perceived learning and (b) satisfaction, such that these relationships will be stronger when conscientiousness is low (versus high).

\subsection{Study 2 Method.}

\subsubsection{Participants}

A total of 151 participants were recruited through MTurk. Inclusion criteria were that individuals must be from the United States, have access to a laptop or desktop computer, have at least a 95\% approval rating within the MTurk system, and have completed at least 100 MTurk HITs. One participant had technical difficulties, and therefore could not get the video to play. Therefore, that participant was removed from the dataset, resulting in a final usable sample of 150 participants. The average age was 34.7 and $45.3 \%$ of the sample was female. In terms of education, $89.3 \%$ of the sample had at least some college experience.

\subsubsection{Measures}

Conscientiousness was measured with 10 items from the International Personality Item Pool (IPIP; Goldberg, 1999). Participants rated the degree to which they agreed that the statement described their personality $(1=$ Strongly disagree; 5 = Strongly agree $)$. Sample items from this scale are "Am always prepared" and "Pay attention to details". This scale demonstrated acceptable internal consistency reliability $(\alpha=.81)$.

Extraversion was measured with 10 items from the IPIP; Goldberg, 1999). Participants rated the degree to which they agreed that the statement described their personality $(1=$ Strongly disagree; 5 = Strongly agree). Sample items from this scale are "Make friends easily" and "Am skilled in handling social situations". This scale demonstrated acceptable internal consistency reliability $(\alpha=.85)$. 
Social presence was assessed with the same scale as in Study 1. This scale demonstrated acceptable internal consistency reliability in the current study, with a Cronbach's alpha $(\alpha)$ value of .89 .

Satisfaction was measured with 5 items from Briggs, Reinig, and de Vreede (2008). Participants rated the degree to which they agreed with each item $(1=$ Strongly disagree; $5=$ Strongly agree). Sample items from this scale are "This online environment gave me a feeling of satisfaction" and "When I think about the online environment, I feel positively toward it". Cronbach's alpha $(\alpha)$ for this scale was .93.

Perceived learning was measured with 4 items that were developed for the current study. Participants rated the degree to which they agreed with each item $(1=$ Strongly disagree; $5=$ Strongly agree). Sample items from this scale are "I learned a lot from this online video" and “After watching this video, I feel I can have a meaningful conversation about it". Cronbach's alpha $(\alpha)$ for this scale was .72.

\subsubsection{Procedure}

Upon agreement to participate in the study, each participant first completed an online survey about their personality (i.e., conscientiousness and extraversion). Then, each participant watched a brief 4.5 minute educational video about the science of sleep. This video was embedded within the onQ platform. Each participant had access to the same video link, and therefore each participant could see and add to the comments and emojis of previous participants. This means that the first participant saw no existing comments and emojis, and the comments/emojis grew with each subsequent user. 
Following the video, participants completed one more brief online survey about their experience with the video (i.e., perceived learning, satisfaction). In exchange for their participation, each participant was compensated with $\$ 2.00$ through the MTurk system.

\subsection{Study 2 Results}

Table 1 presents descriptive statistics, internal consistency reliability coefficients, and zero order correlations among study variables. Note that we also ran partial correlations in which we controlled for user order (to partial out any variance due to the fact that users were exposed to different numbers of comments and emojis from others, depending on the order in which they viewed the video), but controlling for order had very little effect on the correlations. Upon examination of the correlation matrix, results show that social presence was significantly and positively related to both perceived learning $(r=.32, p<.01)$ and satisfaction $(r=.53, p<.01)$, which is in alignment with previous research (e.g., Richardson et al. 2017). Further, user order was correlated with social presence perceptions $(r=.19, p<.05)$, suggesting that as user order increased (and consequently saw more comments and emojis from others), perceptions of social presence increased as well.

Table 1. Descriptive Statistics and Partial Correlations for Study 2

\begin{tabular}{lcccccc}
\hline & 1 & 2 & 3 & 4 & 5 & 6 \\
\hline 1. Social Presence & $.89)$ & & & & & \\
2. Extraversion & .09 & $(.85)$ & & & & \\
3. Conscientiousness & .06 & $.24^{* *}$ & $(.81)$ & & & \\
4. Perceived Learning & $.32^{* *}$ & .12 & $.19^{*}$ & $(.72)$ & & \\
5. Satisfaction & $.53^{* *}$ & $.30^{* *}$ & .07 & $.44^{* *}$ & $(.93)$ & \\
6. User Order & $.19^{*}$ & -.06 & -.05 & .06 & .11 & - \\
\hline$M$ & 3.73 & 2.89 & 3.68 & 3.98 & 3.60 & - \\
$S D$ & 0.92 & 0.81 & 0.63 & 0.65 & 0.96 & - \\
\hline
\end{tabular}

Notes. $N=150 .{ }^{* *} \mathrm{p}<.01,{ }^{*} \mathrm{p}<.05$. Alpha reliability coefficients on diagonal. 
Hypothesis 1, which proposed that extraversion would moderate the relationship between social presence and (a) perceived learning and (b) satisfaction, was tested with moderated regression. First, user order (included as a control) was entered at step one, and then social presence and extraversion were centered and then entered at step 2. Finally, the product of social presence and extraversion was entered at step 3. Results are displayed in Table 2. Hypothesis 1 was not supported, as extraversion did not moderate the relationships between social presence and either outcome.

Table 2. Moderated Regression Results for Extraversion for Study 2

\begin{tabular}{lcc}
\hline & Perceived Learning & Satisfaction \\
\hline Extraversion & & \\
\hline Step 1 & & \\
\hline Order & .09 & .11 \\
$\mathrm{R}^{2}$ & .01 & .01 \\
\hline Step 2 & .04 & \\
\hline Order & $.30^{* *}$ & .03 \\
Social Presence & .10 & $.50^{* *}$ \\
Extraversion & .11 & $.25^{* *}$ \\
$\mathrm{R}^{2}$ & $.10^{* *}$ & .34 \\
$\Delta R^{2}$ & $8.17^{* *}$ & $.33^{* *}$ \\
$\Delta F$ & & $36.07^{* *}$ \\
\hline Step 3 & .04 & \\
\hline Order & $.30^{* *}$ & .04 \\
Social Presence & .10 & $.50^{* *}$ \\
Extraversion & -.09 & $.26^{* *}$ \\
Social Presence x Extraversion & .12 & -.08 \\
$\mathrm{R}^{2}$ & .01 & .35 \\
$\Delta R^{2}$ & 1.43 & .01 \\
$\Delta F$ & & 1.51 \\
\hline Notes $N=150^{* *} p<.01{ }^{*}{ }^{*}<<.05$. &
\end{tabular}

Notes. $N=150 .{ }^{* *} \mathrm{p}<.01,{ }^{*} \mathrm{p}<.05$.

Hypothesis 2, which proposed that conscientiousness would moderate the relationship between social presence and (a) perceived learning and (b) satisfaction, was also tested with moderated regression. As before, user order (included as a control) was entered at step one, and 
then social presence and conscientiousness were centered and then entered at step 2. Finally, the product of social presence and conscientiousness was entered at step 3. Results are displayed in Table 3. Conscientiousness moderated the relationships between social presence and (a) perceived learning $\left(\Delta \mathrm{R}^{2}=.04, p<.05\right)$, as well as (b) satisfaction $\left(\Delta \mathrm{R}^{2}=.03, p<.05\right)$.

Table 3. Moderated Regression Results for Conscientiousness for Study 2

\begin{tabular}{lcc}
\hline & Perceived Learning & Satisfaction \\
\hline Conscientiousness & & \\
\hline Step 1 & & \\
\hline Order & .09 & .11 \\
$\mathrm{R}^{2}$ & .01 & .01 \\
\hline Step 2 & .04 & \\
\hline Order & $.29^{* *}$ & .01 \\
Social Presence & $.21^{* *}$ & $.52^{* *}$ \\
Conscientiousness & .14 & .05 \\
$\mathrm{R}^{2}$ & $.13^{* *}$ & .28 \\
$\Delta R^{2}$ & $11.36^{* *}$ & $.27^{* *}$ \\
$\Delta F$ & & $26.76^{* *}$ \\
\hline Step 3 & .03 & \\
\hline Order & $.34^{* *}$ & .00 \\
Social Presence & $.21^{* *}$ & $.57^{* *}$ \\
Conscientiousness & $-.20^{*}$ & .05 \\
Social Presence x Conscientiousness & .18 & $-.17^{*}$ \\
$\mathrm{R}^{2}$ & $.04^{*}$ & .30 \\
$\Delta R^{2}$ & $6.31^{*}$ & $.03^{*}$ \\
$\Delta F$ &
\end{tabular}

Notes. $N=150 .{ }^{* *} \mathrm{p}<.01,{ }^{*} \mathrm{p}<.05$.

To further probe these significant interactions, simple slopes were calculated at high $(+1$ standard deviation) and low (-1 standard deviation) levels of the moderator (Aiken \& West, 1991). Results of this analysis show that the relationship between social presence and perceived learning was stronger for those who were low in conscientiousness $(\beta=.54, p<.01)$ than those who were high in conscientiousness ( $\beta=.16, p=$ n.s.). Therefore, Hypothesis $2 \mathrm{a}$ was supported. Further, the relationship between social presence and satisfaction was stronger for those who 
were low in conscientiousness $(\beta=.73, p<.01)$ than those who were high in conscientiousness $(\beta=.40, p<.01)$. As such, Hypothesis $2 \mathrm{~b}$ was supported. See Figures 3 and 4 for graphical representations of these moderation effects.

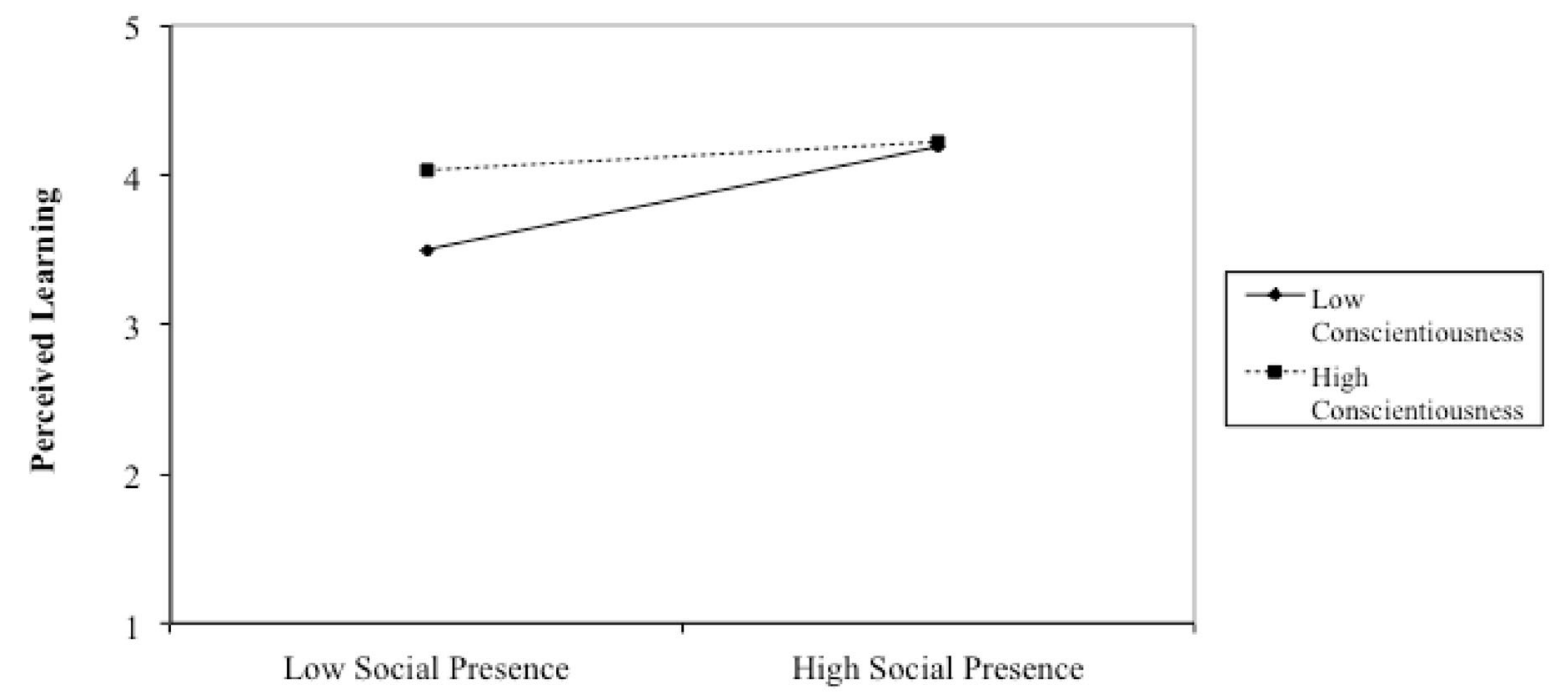

Figure 3. Graphical representation of conscientiousness (+/- 1 standard deviation) as a moderator of the relationship between social presence and perceived learning

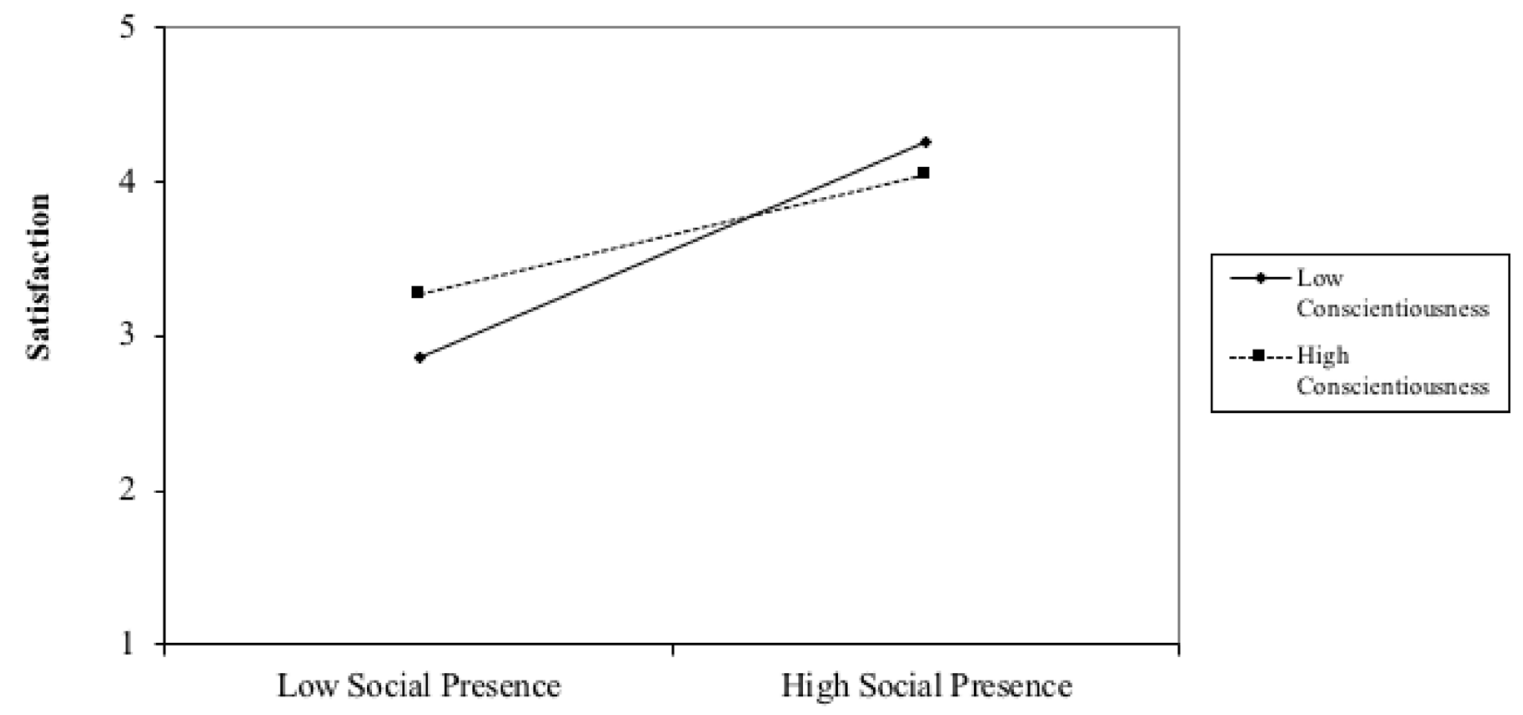

Figure 4. Graphical representation of conscientiousness (+/- 1 standard deviation) as a moderator of the relationship between social presence and satisfaction 


\subsection{Study 2 Brief Discussion}

The aims of the second study were to further validate the use of asynchronous social features to increase social presence perceptions by assessing the relationships between social presence and user outcomes (i.e., perceived learning, satisfaction), and to examine the degree to which personality influences these relationships. We were able to replicate the established relationships between social presence and perceived learning and satisfaction, thus further validating our approach to increase social presence among users. Further, results demonstrated that social presence needs may vary by individual, as social presence was more beneficial for some individuals (e.g., those low in conscientiousness) than for others (e.g., those high in conscientiousness).

\section{Discussion}

As the popularity of online learning continues to increase, so too does the need to understand how to enhance the learning experience for users in online, video-centric environments. The current paper described two studies that contributed to our understanding of this issue in at least three ways.

First, in Study 1 we showed that incorporating asynchronous social features (such as comments) that are embedded onto a video timeline can increase users' social presence perceptions, thereby enhancing their online experience. This effect was found even over a short timeframe of a few minutes, differing from the vast majority of existing research that considers social presence over longer durations (e.g., a semester), and therefore adding to our understanding of how to facilitate social presence perceptions in online classrooms that incorporate a series of short (e.g., 6-9 minute) video lectures (Guo et al., 2014). This is noteworthy, given that up to this point, the existing literature has largely considered social 
presence to be a construct that develops slowly over long periods of time (e.g., over the course of a semester; Akyol \& Garrison, 2008). Parallel to the empirically supported concept of "swift trust", in which individuals quickly develop trust in brief, temporary situations (e.g., Meyersen, Weick, \& Kramer, 1996; Crisp \& Jarvenpaa, 2013), our results provide evidence for the concept of "swift social presence", in which individuals quickly develop social presence perceptions over short, temporary learning experiences.

Further, it is important to note that these comments increased social presence perceptions among users, departing from the majority of social presence intervention work that focuses upon ways to increase social presence between users and instructors (e.g., Dunlap \& Lowenthal, 2010; Duvall et al., 2007; Lowenthal \& Dunlap, 2011). While this past research has provided pertinent insights, there has remained a dearth of research that considers effective ways to facilitate social presence among users. This is surprising, as the Community of Inquiry Framework (Garrison, Anderson, \& Archer, 1999), a leading theory in the social presence literature, proposes that both instructor presence and user social presence (in addition to cognitive presence) are necessary in order to create an optimal educational experience. Ultimately, this study adds to the social presence literature by considering social presence among users, showing that asynchronous video commenting features serve as one feasible way that instructors can incorporate social connection and ultimately social presence perceptions among users within the online learning environment.

Second, in alignment with previous research (e.g., Richardson et al. 2017), in Study 2 we showed that social presence perceptions were associated with positive outcomes, namely perceived learning and satisfaction. This result not only replicates the findings of previous studies, but also adds to existing work, as we considered these associations in the context of a novel online video platform that allows asynchronous communication among users. Ultimately, 
these results show that when we directly examine the continuous relationship between social presence and each outcome of interest within the video-centric environment, social presence perceptions are in fact associated with better outcomes.

Third, and perhaps most importantly, we found evidence in Study 2 that personality is a moderator of the relationships between social presence and both satisfaction and perceived learning, signifying that social presence is particularly important for some individuals (e.g., those low in conscientiousness) than for others. Specifically, social presence was not associated with satisfaction nor perceived learning for conscientious users, suggesting that conscientious users will benefit from an online learning environment, regardless of whether not they perceive high degrees social presence. However, for those low in conscientiousness, social presence was associated with greater perceived learning and satisfaction. This result suggests that social presence serves to "boost" the online experience for these users, and may even compensate for low levels of conscientiousness. These results point to the possibility that social presence interventions, such as the incorporation of asynchronous video comments, may be particularly useful for classes that consist of students who tend to fall lower on the conscientious personality dimension. Further, it is important to note that while some students may gain more from social presence than others, there was no evidence that social presence hurts the student online experience. Ultimately, these results speak to the need of considering students' individual needs when it comes to online learning, and therefore align with recent assertions (e.g., Lowenthal \& Dunlap, 2018; Lowenthal \& Mulder, 2017) that social presence needs vary among individuals.

\subsection{Limitations and Future Directions}

While this research contributes greatly to the online learning literature, it is not without limitations. For instance, all measures in these studies were self-reported, and thus it is possible 
that common-method bias influenced our results. However, it should be noted that commonmethod variance does not tend to produce significant moderation effects (Siemsen, Roth, \& Oliveira, 2010), thus largely assuaging this concern. Regardless, future research should incorporate objective metrics and/or collect self-reported metrics over multiple timepoints in order to validate these findings (Podsakoff, MacKenzie, Lee, \& Podsakoff, 2003).

Additionally, both studies were conducted within a single online learning platform, onQ. This may influence the generalizability of findings to other online platforms beyond onQ. Future research studies should aim to replicate the findings, such as the personality moderation effects, in other online learning contexts and environments in order to assess the generalizability of the findings from the current study.

Similarly, these studies were conducted on MTurk, and therefore participants may have been motivated to participate largely due to the external reward (i.e., monetary compensation), and thus may not have been as intrinsically motivated to learn as students who are officially enrolled in an online course. This may impact the generalizability of our findings to other individuals outside of MTurk. Therefore, future research should replicate these results in an established online course, such as a massive open online course (MOOC) or a universitysponsored online class. Future research should also consider other personal factors (e.g., participants' age, levels of digital literacy) and contextual factors (e.g., length of course) that may influence social presence perceptions in an online context, and may also consider linking social presence to additional important outcomes, such as actual learning and student engagement.

Further, it should be noted that the experiment in Study 1 only included two conditions, (i.e, seeded and unseeded), thereby limiting the conclusions that can be drawn from that study. 
We encourage future researchers to build upon this work by including more incremental levels into their research designs (e.g., multiple experimental conditions that vary in the number of seeded comments that are presented to participants).

In terms of additional directions for future work, this research has implications for the design of online learning platforms in video-centric environments and for the development of new theory. While any new design will need to be experimentally validated, two key possibilities to explore include (a) providing users with more control on how social features impact their experience, e.g. adding the ability to "turn up or down" the social chatter and (b) developing personalized variants of the platform. Ultimately, building upon this stream of research will allow for the development of a new theory to describe and explain the differential impact of social presence among users, allowing researchers to understand for whom social presence is particularly valuable, and perhaps when as well. These are all promising and significant directions for future work that can significantly enhance the digital learning platforms of today that are only going to grow in the years ahead.

\subsection{Conclusion}

Results of these current studies suggest that asynchronous social features (i.e., videotagged commenting) serve as a feasible way to facilitate social presence among users in an online, video-centric learning environment. Further, we showed that the degree to which users gain benefits from social presence differ depending on personal characteristics (i.e., personality). Ultimately, this research provides important insights into the influence and importance of social presence among users within online learning environments. 


\section{References}

Aiken, L. S., \& West, S. G. (1991). Multiple regression: Testing and interpreting interactions. Thousand Oaks, CA, US: Sage Publications, Inc.

Akyol, Z., \& Garrison, D. R. (2008). The development of a community of inquiry over time in an online course: Understanding the progression and integration of social, cognitive and teaching presence. Journal of Asynchronous Learning Networks, 12, 3-22.

Bailenson, J. N., Blascovich, J., Beall, A. C., \& Loomis, J. M. (2003). Interpersonal distance in immersive virtual environments. Personality and Social Psychology Bulletin, 29(7), 819833.

Berger, J. B., \& Milem, J. F. (1999). The role of student involvement and perceptions of integration in a causal model of student persistence. Research in Higher Education, 40(6), 641-664.

Borup, J., West, R. E., \& Graham, C. R. (2012). Improving online social presence through asynchronous video. The Internet and Higher Education, 15(3), 195-203.

Borup, J., West, R. E., Thomas, R. A., \& Graham, C. R. (2014). Examining the impact of video feedback on instructor social presence in blended courses. International Review of Research in Open and Distributed Learning, 15(3), 232-256.

Briggs, R. O., Reinig, B. A., \& de Vreede, G. J. (2008). The yield shift theory of satisfaction and its application to the IS/IT domain. Journal of the Association for Information Systems, 9(5), 267-293.

Brown, C. J. (2020). Barnes \& Noble Education survey reveals college student preparedness split: Technically ready for online learning, but emotionally unsure. Retrieved from https://www.businesswire.com/news/home/20200408005156/en/ 
Burke, L. (2020). Colleges move online amid virus fears. Retrieved from https://www.insidehighered.com/news/2020/03/09/colleges-move-classes-onlinecoronavirus-infects-more

Cohen, J. (1988). Statistical power analysis for the behavioral sciences, 2nd ed. Hillsdale, NJ: Erlbaum.

Costa Jr, P. T., \& McCrae, R. R. (1992). Four ways five factors are basic. Personality and Individual Differences, 13(6), 653-665.

Crisp, C. B., \& Jarvenpaa, S. L. (2013). Swift trust in global virtual teams. Journal of Personnel Psychology, 12(1), 45-56.

Dunlap, J., \& Lowenthal, P. (2010, December 15). Defeating the Kobayashi Maru: Supporting Student Retention by Balancing the Needs of the Many and the One. Retrieved March 1, 2019, from https://er.educause.edu/articles/2010/12/defeating-the-kobayashi-marusupporting-student-retention-by-balancing-the-needs-of-the-many-and-the-one

DuVall, J. B., Powell, M. R., Hodge, E., \& Ellis, M. (2007). Text messaging to improve social presence in online learning. Educause Quarterly, 30(3), 24-28.

Garrison, D. R., Anderson, T., \& Archer, W. (1999). Critical inquiry in a text-based environment: Computer conferencing in higher education. The Internet and Higher Education, 2(2-3), 87-105.

Goldberg, L. R. (1999). A broad-bandwidth, public domain, personality inventory measuring the lower-level facets of several five-factor models. Personality Psychology in Europe, 7(1), 7-28.

Goldstein, D. (2020). Coronavirus is shutting schools. Is America ready for virtual learning? Retrieved from https://www.nytimes.com/2020/03/13/us/virtual-learning-challenges.html 
Gunawardena, C. N. (1995). Social presence theory and implications for interaction and collaborative learning in computer conferences. International Journal of Educational Telecommunications, 1(2), 147-166.

Guo, P. J., Kim, J., \& Rubin, R. (2014, March). How video production affects student engagement: An empirical study of MOOC videos. In Proceedings of the first ACM conference on Learning@Scale conference (pp. 41-50). ACM.

Guo, P. J., \& Reinecke, K. (2014, March). Demographic differences in how students navigate through MOOCs. In Proceedings of the first ACM conference on Learning@Scale conference (pp. 21-30). ACM.

Hughes, J. N., Luo, W., Kwok, O. M., \& Loyd, L. K. (2008). Teacher-student support, effortful engagement, and achievement: a 3-year longitudinal study. Journal of Educational Psychology, 100(1), 1-14.

Kear, K., Chetwynd, F., \& Jefferis, H. (2014). Social presence in online learning communities: the role of personal profiles. Research in Learning Technology, 22, 1-15.

Kirkpatrick, D. L. (1959). Techniques for Evaluation Training Programs. Journal of the American Society of Training Directors, 13, 21-26.

Komarraju, M., Karau, S. J., \& Schmeck, R. R. (2009). Role of the Big Five personality traits in predicting college students' academic motivation and achievement. Learning and Individual Differences, 19(1), 47-52.

Kreijns, K., Kirschner, P. A., Jochems, W., \& Van Buuren, H. (2011). Measuring perceived social presence in distributed learning groups. Education and Information Technologies, $16(4), 365-381$. 
Lowenthal, P. R., \& Dunlap, J. (2011, April). Investigating students' perceptions of various instructional strategies to establish social presence. Paper presented at the Annual Meeting of the American Educational Research Association, New Orleans, LA.

Lowenthal, P. R., \& Dunlap, J. C. (2018). Investigating students' perceptions of instructional strategies to establish social presence. Distance Education, 39(3), 281-298.

Lowenthal, P., \& 8, D. (2017). Social presence and communication technologies: Tales of trial and error. In Social presence in online learning: Multiple perspectives on practice and research (pp. 32-44). Sterling, VA: Stylus Publishing.

Lyons, A., Reysen, S., \& Pierce, L. (2012). Video lecture format, student technological efficacy, and social presence in online courses. Computers in Human Behavior, 28(1), 181-186.

Mazzolini, M., \& Maddison, S. (2007). When to jump in: The role of the instructor in online discussion forums. Computers \& Education, 49(2), 193-213.

Meyerson, D., Weick, K. E., \& Kramer, R. M. (1996). Swift trust and temporary groups. In R. M. Kramer \& T. R. Tyler (Eds.), Trust in organizations: Frontiers of theory and research (pp. 166-195). Thousand Oaks, CA: Sage Publications.

Podsakoff, P. M., MacKenzie, S. B., Lee, J.-Y., \& Podsakoff, N. P. (2003). Common method biases in behavioral research: A critical review of the literature and recommended remedies. Journal of Applied Psychology, 88(5), 879-903.

Reio Jr, T. G., \& Crim, S. J. (2013). Social presence and student satisfaction as predictors of online enrollment intent. American Journal of Distance Education, 27(2), 122-133.

Richardson, J. C., Maeda, Y., Lv, J., \& Caskurlu, S. (2017). Social presence in relation to students' satisfaction and learning in the online environment: A meta-analysis. Computers in Human Behavior, 71, 402-417. 
Rourke, L., Anderson, T., Garrison, R., \& Archer, W. (2001). Methodological issues in the content analysis of computer conference transcripts. International Journal of Artificial Intelligence in Education, 12(1), 8-22.

Seaman, J. E., Allen, I. E., \& Seaman, J. (2018). Grade Increase: Tracking Distance Education in the United States. Babson Survey Research Group. Retrieved from https://onlinelearningconsortium.org/read/grade-increase-tracking-distance-educationunited-states/

Short, J., Williams, E., \& Christie, B. (1976). The social psychology of telecommunications. Hoboken, NJ: John Wiley \& Sons.

Siemsen, E., Roth, A., \& Oliveira, P. (2010). Common method bias in regression models with linear, quadratic, and interaction effects. Organizational Research Methods, 13(3), 456476.

Swan, K. (2003). Developing social presence in online course discussions. In S. Naidu (Ed.), Learning and teaching with technology: Principles and practices (pp. 147-164). London: Kogan Page.

Swan, K., \& Shih, L. F. (2005). On the nature and development of social presence in online course discussions. Journal of Asynchronous Learning Networks, 9(3), 115-136.

Trapmann, S., Hell, B., Hirn, J. O. W., \& Schuler, H. (2007). Meta-analysis of the relationship between the Big Five and academic success at university. Journal of Psychology, 215(2), $132-151$

Watson, D., \& Clark, L. A. (1997). Extraversion and its positive emotional core. In R. Hogan, J. Johnson, \& S. Briggs (Eds.), Handbook of personality psychology (pp. 767-793). San Diego: Academic Press. 